\title{
Introducing Uncertainty into Social Simulation: Using Fuzzy Logic for Agent-Based Modelling
}

\section{Samer Hassan*, Luis Garmendia and Juan Pavón}

Universidad Complutense Madrid, Facultad de Informática, Ciudad Universitaria s/n, 28040 Madrid, Spain Fax: +34 913947556 E-mail:samer@fdi.ucm.es E-mail: lgarmend@fdi.ucm.es

E-mail: jpavon@fdi.ucm.es

${ }^{*}$ Corresponding author

\begin{abstract}
Agent based models are useful to study emergent behaviour in social systems. In general, existing agent models tend to be quite simple, but there are social problems that require the consideration of some aspects with uncertainty as human thinking does. Those characteristics can be addressed by using fuzzy sets theory in the specification of the attributes that describe agents representing individuals, and in the functions that model the evolution of individual change of mind, the relationships among individuals in a social network, the inheritance, and the similarity between individuals. This paper discusses the fuzzification of agent-based models and analyses experimentation results for a specific case of the study of the evolution of human values in a society.
\end{abstract}

Keywords: ABSS; agent-based social simulation; ABM; agent based model; complexity in agent based models; EVS; European Values Survey; fuzzy agents; fuzzy logic; fuzzification; MAS; multi-agent system; reasoning-based intelligente system; similarity; social network; uncertainty.

Reference to this paper should be made as follows: Hassan, S., Garmendia L. and Pavón, J. (xxxx) 'Introducing Uncertainty into Social Simulation: Using Fuzzy Logic for Agent-Based Modelling', Int. J. Reasoning-based Intelligent Systems, Vol. x, No. x, pp.xxx-xxx.

\section{Introduction}

Agent based social simulation (ABSS) is applied as a tool for the study of the evolution of social systems. This has proved to be successful for a considerable number of cases, usually involving simple agent models following the KISS ("Keep it simple, stupid") principle proposed by Axelrod (1997) and extended over many well-known examples Gilbert \& Troitzsch (1999). However, some systems cannot 
be easily modelled with just a set of numeric or enumerated variables, and very simple agents, closer to the classical cellular-automata approach. The introduction of increasing complexity is an open debate in the community (e.g. Hassan, Antunes, Pavon \& Gilbert (2008)), and several lines are being explored. Some problems where the agents need complex planification and decision-making may need cognitive models based on psychology (e.g. Conte \& Paolucci (2001)). But others where the key obstacle to overcome is the degree of uncertainty that must be considered constitutes a difficult issue in the field. For instance, we can analyse the evolution of values in human societies. When considering sociological surveys, such as the European Values Survey (2000), which is run periodically, it can be observed that although there is an effort to categorize the possible answers to questions such as "Do you trust political parties?", there is always this mentioned degree of uncertainty. This is even more evident when considering the evolution of values in individuals. This evolution is a consequence of multiple factors, which are strongly intertwined.

In this paper it is proposed the Fuzzy logic as a helpful tool to deal with such kind of approximate or uncertainty knowledge. More specifically, it analyses how fuzzy logic can be useful when building models for ABSS. This analysis comprises different aspects that can be fuzzificated from an agent based social model, so it gets better adapted to reality, and more specifically to facilitate the study of the evolution of values in human societies. In concrete, this work has been applied to model and simulate the evolution of religiosity in European societies, based on a sociological study Menendez (2004). Although this has been implemented on Repast (2008), a well-known ABSS tool, some additions have been made to the existing software library in order to be able to model and operate with fuzzy logic attributes and relationships

The following section discusses the adequacy of agent based modelling for social systems simulation, and introduces some of the characteristics of the system under study, that require the consideration for using fuzzy logic. Section 3 introduces fuzzy logic concepts related to this environment. Section 4 presents each part of the model that is fuzzificated. Finally, the final part summarizes the main results and contributions of this work and some issues for improving this framework.

\section{Modelling and Simulation of Social Systems}

Social phenomena are extremely complicated and unpredictable, since they involve complex interaction and mutual interdependence networks. A social system consists of a collection of individuals that interact among them, evolving autonomously and motivated by their own beliefs and personal goals, and the circumstances of their social environment.

A multi-agent system (MAS) consists of a set of autonomous software entities (the agents) that interact among them and with their environment. Autonomy means that agents are active entities that can take their own decisions. In this sense, the agent paradigm assimilates quite well to the individual in a social system, so it can be used to simulate them, exploring the complexity of social dynamics. With this perspective, agent-based simulation tools have been developed in the last years to explore the complexity of social dynamics Gilbert \& Troitzsch (1999). An agent- 
based simulation executes several agents, which can be of different types, in an observable environment where agents' behaviour can be monitored. Observations on agents can assist in the analysis of the collective behaviour and trends of system evolution. This provides a platform for empirical studies of social systems. As simulation is performed in a controlled environment, on one or several processors, this kind of tools allows the implementation of experiments and studies that would not be feasible otherwise, as Axelrod (1997) stands.

There are, however, some limitations when trying to simulate real social systems. The main issue is that the real individual, with regard to a software agent, is by itself a complex system, whose behaviour is unpredictable and less determined than for an agent, whose behaviour and perception capabilities can be designed with relative simplicity. Moreover, it is not possible in practice to consider the simulation of countless nuances that can be found in a real social system with respect to agent interaction, characterization of the environment, etc. For this reason, it is impractical to intend the simulation of a social system in all dimensions. On the other hand, we should limit to simulate concrete social processes in a systemic and interactive context. Therefore, the simulation of social systems should be considered in terms of focus on a specific process.

In spite of these limitations, the agent paradigm offers many advantages to express the nature and peculiarities of social phenomena. However, existing agent based simulation tools promote the use of rather simple agent models, basically as a kind of cellular automata Wolfram (2002). This may be valid to study emergent behaviour that results from deterministic behaviour of agents. But, as it has been already explained, when considering the evolution of complex mental entities, such as human believes and values, that approach is rather limited.

As an example of a system that requires further considerations on agent modelling, think about some sociological analysis derived from the European Value Survey and of the World Value Survey by Norris \& Inglehart (2004). In these surveys there are many questions about the degree of happiness, satisfaction in different aspects of life, or trust in several institutions. Although there is some kind of categorization for the possible answers, such as "Very much" or "Partially", there is always some degree of imprecision, which is difficult to model with discrete/crisp categories. Even more, when the individual is evolving to different positions, some of these values get even more undefined. This issue arises also when modelling agent relationships such as friendship: is it possible to measure a degree of friendship between two persons? Agent fuzzification has been already applied for social network analysis, as in the work of Carbó, Molina and Dávila (Carbó et al. (2005)), but considering mainly relationships among agents. Here we are considering five aspects of the multi-agent system: agent attributes, functions for similarity and evolution of agent behaviour, relationships among agents in social networks, and inheritance.

\section{Fuzzy Logic in this Context}

Fuzzy logic is useful in environments with vague and uncertain information and reasoning Zadeh (1965), as it has been discussed above for some aspects in the study of human societies. In the modelling of ABSS, we have counted with the help of 
an expert in Sociology, who has been consulted repeatedly along the process, and frequently he uses linguistic variables and terms to express his knowledge that can be better represented with fuzzy sets.

Given a universe of discourse $\mathrm{U}$, a fuzzy set $\mu \mathrm{U} \rightarrow[0,1]$ on $\mathrm{U}$ is a mapping that gives a membership degree in the interval $[0,1]$ to every element of $U$. Note that classical sets are particular cases of fuzzy sets. There are many human characteristics that have not a clear boundary or depend on the interpretation or context, as tall or beauty, that can be represented by a fuzzy set on the set of human persons, and gives a membership degree of the characteristic, that can be, for example 0.8.

Given some fuzzy sets representing characteristics, sometime it is needed to model algebraical operations on them, as 'tall and beauty', that must also be modeled, for example to fire a rule in approximate reasoning systems. We need to use different operators to define those algebras, or logics, generalizing the classical intersection, union and complement of classical sets.

A binary operator $\mathrm{T}:[0,1] \times[0,1] \rightarrow[0,1]$ is a triangular norm (t-norm) (Klement et al. (2000), Schweizer \& Sklar (2005)) if it satisfies the following axioms:

1. $T(1, x)=x$

2. $\mathrm{T}(\mathrm{x}, \mathrm{y})=\mathrm{T}(\mathrm{y}, \mathrm{x})$

3. $\mathrm{T}(\mathrm{x}, \mathrm{T}(\mathrm{y}, \mathrm{z}))=\mathrm{T}(\mathrm{T}(\mathrm{x}, \mathrm{y}), \mathrm{z})$

4. If $\mathrm{x} \leq \mathrm{x}^{\prime}$ and $\mathrm{y} \leq \mathrm{y}^{\prime}$ then $\mathrm{T}(\mathrm{x}, \mathrm{y}) \leq \mathrm{T}\left(\mathrm{x}^{\prime}, \mathrm{y}^{\prime}\right)$.

The t-norm operators can be used to define the intersection of two fuzzy sets $\mathrm{A}$ and $\mathrm{B}$, as follows: $\mu_{A \cap B}(x)=T\left(\mu_{A}(x), \mu_{B}(x),\right)$ for all $\mathrm{x}$ in $\mathrm{U}$. In a similar way, the union and complements of fuzzy sets can be defined using triangular conorms (again Klement et al. (2000), Schweizer \& Sklar (2005)) and negation operators, which define different fuzzy logics. The most used fuzzy logics in applications use continuous t-norms and t-conorms, as the Zadeh logic, from Zadeh (1965), that uses the minimum t-norm and maximum t-conorm to generalize the AND and OR classical logic operators, the product logic, and the Lukasiewicz logic.

Fuzzy relations $\mathrm{R}$ : $\mathrm{UxU}([0,1]$ have many applications to represent degrees of relations between objects in an universe. Those relations can also be characterized without a nitide border, as the friendship relation. Classical relations cannot express some types of information as 'we are more or less friends', or 'a little bit friends'. Fuzzy relations are mostly used in Artificial Intelligence applications to represent degrees of similarity (that define not nitide groups or clusters) or to model implications rules to make inference with uncertainty, imprecision or lack of knowledge.

The classical concept of transitivity is generalised in fuzzy logic by the Ttransitivity property of fuzzy relations.

Let $\mathrm{T}$ be a t-norm. A fuzzy relation $\mathrm{S}$ is $\mathrm{T}$-transitive if $\mathrm{T}(\mathrm{S}(\mathrm{a}, \mathrm{b}), \mathrm{S}(\mathrm{b}, \mathrm{c})) \leq$ $\mathrm{S}(\mathrm{a}, \mathrm{c})$ for all a, b, c in E. A T-indistinguishability (as in Valverde (1984)) fuzzy relation $\mathrm{S}$ on a universe $\mathrm{E}$ is a mapping $\mathrm{S}: \mathrm{ExE} \rightarrow[0,1]$, satisfying the reflexivity, symmetry and T-Transitivity properties. A similarity relation, Zadeh (1971), is a Min-indistinguishability.

The similarity fuzzy relations generalise the classical equivalence relations that are so useful to define partitions in a set. 
The $\mathrm{T}$-transitive closure $\mathrm{R}^{T}$ of a fuzzy relation $\mathrm{R}$ is the lowest relation that contains $\mathrm{R}$ and is T-transitive. Of course, it generalises the classical transitive closure when all degrees are in $\{0,1\}$. There are many proposed algorithms to compute the T-transitive closure: Naessens et al. (2002).

An algorithm used to compute the T-transitive closure $\mathrm{R}^{T}$ of a fuzzy relation $\mathrm{R}$ is the following:

1. $\mathrm{R}^{\prime}=\mathrm{R} \cup_{M a x}\left(\operatorname{Ro}_{S u p-T} \mathrm{R}\right)$

2. If $\mathrm{R}^{\prime} \neq \mathrm{R}$ then $\mathrm{R}:=\mathrm{R}^{\prime}$ and go back to 1 ), otherwise stop and $\mathrm{R}^{T}:=\mathrm{R}$ '.

In this work, we define fuzzy sets and fuzzy relations on the universe of individuals $\mathrm{U}=\left\{\right.$ Individual $_{i=1 . . N}$ to represent different characteristics, linguistic variables and relations between persons.

\section{Fuzzification of Agent Model}

\subsection{Fuzzy Agent Relationships}

The analysis of the evolution of values in a human society is not a trivial case for agent based modelling. This has been already studied in a previous work in Pavón et al. (2008) by considering the evolution of religiosity in Spanish society, as there is considerable research on this issue in the domain of Sociology: Menendez (2004). The simulation of the model has proved alignment with survey data (from the European Value Survey, which runs periodically each 10 years). However, abstractions for the agent model are in some aspects over-simplistic, and do not allow the social scientist to interpret results in the same terms that the survey data are expressed. Furthermore, some simplifications produce some divergences of results with respect to survey data. These facts have motivated the use of fuzzy logic to get agent models that are closer to real system. In this way, agent models have been enriched with the use of fuzzy logic in five aspects: relationships among agents, some variable attributes that determine agent states, functions of similarity, evolution of agent states, and inheritance.

The cited multi-agent system of Pavón et al. (2008) defines two possible relationships among individuals (agents): friendship and family. Friendship is naturally predisposed to be fuzzyfied because if the relation friendship is defined as a Boolean, two individuals can only be or not to be friends, which is unrealistic in real life. Therefore, it is a closer model to represent this relation using a continuous range of grades of friendship. Therefore, friendship is defined as a fuzzy relation, with real friendship degree between 0 and 1 for every couple of individuals.

Let $\mathrm{R}_{\text {friend }}: \mathrm{UxU} \rightarrow[0,1]$ be the fuzzy relation on the set of individuals that give a degree of "friendship". This fuzzy relation gives a degree of friendship in the interval $[0,1]$ for every couple on individuals. Let Ind be an individual in $U$. The classic set Friends (Ind) is defined as the set of all the individuals $\mathrm{x}$ in $\mathrm{U}$ whose $\mathrm{R}_{\text {friend }}(\mathrm{Ind}, \mathrm{x})$ is greater than 0 .

And so every individual will have a range from true close friends to just "known" people with the rest of individuals. Of course, some restrictions to this definition could be introduced in order to suit context needs. 
The 'family link' relation is also fuzzificated by following the condition that this relationship would be "1" in direct family (parents, brothers), lower with further familiars, and "0" with people that is not family at all. Thus, the 'family link' characteristic on couples of individuals is modeled with the $\mathrm{R}_{\text {family }}: \mathrm{Ux} \mathrm{U} \rightarrow[0,1]$ fuzzy relation.

\subsection{A fuzzy method to simulate matchmaking}

The social system also simulates matchmaking. Once a couple is made (for example, a marriage), a stable couple can be defined as a crisp relation: two persons are a couple or are not. We propose to learn this classical relation $\mathrm{R}_{\text {couple }}: \mathrm{UxU} \rightarrow$ $\{0,1\}$ by using approximate reasoning and fuzzy inference techniques.

Of course, if we know a $\mathrm{R}_{\text {couple }}$ relation, we also know a 'single' and 'married' classical set on $\mathrm{U}$, defined as:

$$
\begin{aligned}
& \text { 'married }(\text { ind })= \begin{cases}1, & \exists \text { ind } 2 / R_{\text {couple }}(\text { ind }, \text { ind } 2)=1 \\
0, & \text { otherwise }\end{cases} \\
& \text { 'simple }(\text { ind })=N O T^{\prime} \text { 'married' }(\text { ind })
\end{aligned}
$$

It does not exist a specific process for finding a couple in previous social simulation models. We decided to ask our sociology expert and build up a general definition for it. An agent will find a couple (always with a random possibility of failure not defined in the mathematical definitions) between the friends that have different sex, are adults and have not yet a couple. The chosen one will be the "most compatible" one, where compatibility is defined as the aggregation of how friends are they and how similar are they (we know that this is an oversimplification with respect to real life, and it is further work to allow some randomness in this process).

This important information of how couples can me made can be obtained by infering a fuzzy relation $\mathrm{R}_{\text {compatible }} \mathrm{UxU} \rightarrow[0,1]$, which can be obtained using a fuzzy aggregation operator, Yager (1993), and operations on the classical set 'has not couple': $\mathrm{U} \rightarrow\{0,1\}$, the fuzzy set 'adult': $\mathrm{U} \rightarrow[0,1]$, the classical relation 'has different sex':UxU $\rightarrow\{0,1\}$, and one fuzzy rule of inference, where the premise is the conjunction of the classical sets.

An Ordered Weighted Averaging (OWA) (see Yager (1993)) is a family of multicriteria combination (aggregation) procedures. By specifying suitable order weights (whose sum will result always 1 ) it is possible to change the form of aggregation: for example, to get the arithmetic average, give the value 0.5 to both weights of the OWA. We can formally define the $\mathrm{R}_{\text {compatible }}: \mathrm{UxU} \rightarrow[0,1]$ fuzzy relation using the $\mathrm{R}_{\text {similarity }}: \mathrm{UxU} \rightarrow[0,1]$ and the $\mathrm{R}_{\text {friend }}: \mathrm{UxU} \rightarrow[0,1]$ fuzzy relation as the following mapping:

$\mathrm{R}_{\text {compatible }}\left(\right.$ Ind, Ind2) $:=$ OWA $\left(\mathrm{R}_{\text {friend }}(\right.$ Ind, Ind2 $), \mathrm{R}_{\text {similarity }}($ Ind, Ind2) $)=$ $\mathrm{w}_{1} * \mathrm{R}_{\text {friend }}$ (Ind, Ind2) $+\mathrm{w}_{2} * \mathrm{R}_{\text {similarity }}$ (Ind,Ind2) for all Ind, Ind2 in U, where $\mathrm{w}_{1}+\mathrm{w}_{2}=1$.

After computing the $\mathrm{R}_{\text {compatible }}$ fuzzy relation, a $\mathrm{R}_{\text {'couplecandidate' }}: \mathrm{UxU} \rightarrow[0$, 1] fuzzy relation can be computed by using the 'single' set and the 'Adult' fuzzy set on $\mathrm{U}$ as follows: 
$\mathrm{R}_{\text {'couple-candidate' }}$ (Ind, Ind2) := Adult(Ind) AND Adult(Ind2) AND 'single'(Ind)

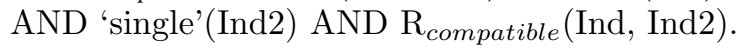

Note that the AND conjunction is implemented by a t-norm. It can be done many times because of the associative property of t-norms. So $\mathrm{T}(\mathrm{x}, \mathrm{y}, \mathrm{z})=\mathrm{T}(\mathrm{T}(\mathrm{x}$, y), z).

Then, the method to marry an individual Ind is to find Ind2 such that it maximizes the fuzzy relation $\mathrm{R}_{\text {'couple-candidate' }}$ (Ind, Ind2). Then it is possible to compute $\mathrm{R}_{\text {couple }}($ Ind, Ind2) $:=1$.

\subsection{Measuring the Similarity between two Agents}

To compute the fuzzy relation $\mathrm{R}_{\text {compatible }}$, used to marry individuals, we have used a fuzzy relation $\mathrm{R}_{\text {similarity }} \mathrm{UxU} \rightarrow[0,1]$ that deserves a deeper study. In the original MAS, the similarity was modelled and implemented through a not normalized crude gratification algorithm, based on the amount of points gathered from the comparison of the agents' crisp attributes. By defining fuzzy sets over these variables (the attributes) and fuzzificating the similarity operator based on them we will be able to have much more accuracy in the determination of similarity between individuals. Moreover, with those fuzzy set we will be able to make inference based on them.

Therefore, we continue defining these fuzzy sets based on the variables that define each individual. Even though there are some of them that are not fuzzificable, like the sex, the most part of them will let us define a fuzzy set over them. For example: Let $\mu_{\text {religious }}: \mathrm{U} \rightarrow[0,1]$ be the fuzzy set that gives a religious grade based on the religiosity variable of the individual. This fuzzy set can be defined by segments with different growth (orthodox people, moderated religious ones, agnostics, atheists...) or by a linear function. Thus, $\mu_{\text {religious }}(\mathrm{Ind})=0.2$ would mean that this person is mainly not religious. The same way we could continue with other fuzzy sets.

Now, with fuzzy sets over the attributes, we can define the fuzzy similarity using a T-indistinguishability, which generalizes the classical equivalence relations. It can be obtained from the negation of a $\mathrm{T}^{*}$-distance, where $\mathrm{T}^{*}$ is the dual t-conorm of the t-norm $\mathrm{T}$. This way, by aggregating the normal distance of each couple of fuzzy sets, allows to obtain the total similarity between two individuals by using a strong negation Schweizer \& Sklar (2005) operator N, a set of fuzzy sets M representing human characteristics, a distance d and an OWA operator, as follows:

$$
R_{\text {similarity }}(\operatorname{Ind}, \operatorname{Ind} 2)=O W A\left(\forall \mu_{i} \text { in } \mathrm{M}, N\left(d\left(\mu_{i}(\operatorname{Ind}), \mu_{i}(\operatorname{Ind} 2)\right)\right)\right.
$$

\subsection{The Influence as the result of the interaction among Agents}

We can focus now on the direct interaction between the agents. In this system, there are some attributes that influence each other. Some attributes, such as sex or age are not affected by other attributes, but some, as ideology, are influenced by the political trends of friends. This local influence is, by definition, a "fuzzy concept": the influence on a person cannot be easily quantified. But after a deep 
analysis and with the help of the sociological expert, we can dare this mathematical definition:

Let $\mathrm{X}$ be a fuzzy set on $\mathrm{U}$ expressing a human characteristic.

The fuzzy set "variation of the attribute "X") on $\mathrm{U}$, denoted $\Delta^{X}: \mathrm{U} \rightarrow[0,1]$ determining the influence of the characteristic $\mathrm{X}$ by the environment of each individual "Ind" can be defined as the aggregation of the influence of all its relatives, friends and couple. This influence is determined by the "proximity" of the person, the distance d between the attribute selected, and how young "Ind" is (if the agent is younger, it will be more receptive to be influenced).

$\Delta^{X}(\mathrm{Ind})=$ OWA $_{i=1 \ldots N}\left(\mathrm{R}_{\text {proximity }}\left(\operatorname{Ind}_{1} \operatorname{Ind}_{i}\right)\right.$ AND d(X(Ind) $\left.-\mathrm{X}\left(\operatorname{Ind}_{i}\right)\right)$ AND $\mu_{\text {young }}($ Ind $\left.)\right)$.

We use the product t-norm to compute the 'AND' conjunction.

Let $\mathrm{R}_{\text {proximity }}\left(\operatorname{Ind}_{n}, \operatorname{Ind}_{i}\right): \mathrm{UxU} \rightarrow[0,1]$ be the fuzzy relation on the set of individuals that give a degree of "proximity". This fuzzy relation is defined by the aggregation (OWA) of the classical relation "couple" with the fuzzy relations $\mathrm{R}_{\text {friend }}$ and $\mathrm{R}_{\text {family: }}$

$\mathrm{R}_{\text {proximity }}$ (Ind, Ind2) := OWA ( $\mathrm{R}_{\text {couple }}\left(\mathrm{Ind}\right.$, Ind2), $\mathrm{R}_{\text {friend }}\left(\mathrm{Ind}\right.$, Ind2), $\mathrm{R}_{\text {family }}$ (Ind, Ind2) $)=\mathrm{w}_{1} * \mathrm{R}_{\text {couple }}\left(\right.$ Ind, Ind2) $+\mathrm{w}_{2} * \mathrm{R}_{\text {friend }}\left(\right.$ Ind, Ind2) $+\mathrm{w}_{3} * \mathrm{R}_{\text {family }}$ (Ind, Ind2)

And of course, the evolution of an attribute is determined, by each individual, as $\mathrm{X}(\mathrm{Ind}):=\mathrm{OWA}\left(\mathrm{X}(\mathrm{Ind}), \Delta^{X}\right.$ (Ind))

\subsection{Other applications}

With continuous influence the global average of the variables will change over time. But there is another source of change: demographic evolution. As time steps go on, agents will find couples and have offspring. Those descendants must inherit their parents' characteristics in some way. In the crisp MAS we solve this problem in a rough way: we obtain the new variables from the average of the parents. But with the use of fuzzy sets on those variables, it is possible to use fuzzy operators between them. Thus, we decided to use the fuzzy connectives $o$ for obtaining the variables of the new individuals (mixed with a random mutation factor not included in the mathematical definition, which was introduced in favour of diversity):

$\forall \mathrm{X}$ attribute of Ind, $\mu_{x}\left(\right.$ Ind) $=\mu_{x}$ (Father (Ind)) o $\mu_{x}$ (Mother (Ind))

Another important side of the agents is their states. The state of an agent is defined by its age, and determines its behaviour. Therefore, an agent in the state of "child" cannot influence adults and cannot find a stable couple, while an "old" agent will not have children and will have greater probability of dying. But, where are the limits between states? In the crisp systems, there are threshold limits that determine that change. For example, if an agent has an age over 27 it is in the "adult" state, but if it is under it, it is in "young" state with a radical different behaviour. This is not realistic: in reality the changes are gradual, as people get older. So, this is another case for applying fuzzy logic again, even though this time is quite difficult: it is easy to define how young is an individual, but it is difficult 
to change gradually its behaviour (anyway, it is an implementation problem, which will not be analysed here).

The last improvement we propose is for extracting new knowledge from the system, using a fuzzy operation: the T-transitive closure. The repeatedly application of transitive property of a relationship facilitates the discovery of the influence of some variables in other "far" ones (the multiple paths of different length between those variables). This operation suits perfectly with the natural transitivity of the (fuzzy) friendship relation: the friend of my friend is not very friend of me. In this way it is possible to infer inferior friendship grades in others. For instance, if $\mathrm{A}$ and $\mathrm{B}$ are friends in a 0.8 , and $\mathrm{B}$ and $\mathrm{C}$ in 0.6 , it can be deduced that $\mathrm{A}$ and $\mathrm{C}$ are friends in a 0.48 . This could continue inferring how an agent $\mathrm{D}$ is friend of $\mathrm{A}$, and so on. We are extracting new knowledge from the system, which would be impossible with a crisp relation for friendship (we cannot infer that A and C are automatically $100 \%$ friends).

We have imposed T-transitivity (e.g. Garmendia et al. (1999)) to the 'friendship' fuzzy relation as a learning process of friendship of individuals with a common friend, and also as a coherence method.

The Zadeh's logic (which uses the t-norm minimum) works well, but we did not choose it because the friendship of $\mathrm{A}$ and $\mathrm{C}$ is either the friendship of $\mathrm{A}$ and $\mathrm{B}$ or the friendship of $\mathrm{B}$ and $\mathrm{C}$, and there is no reason for that. $\mathrm{A}$ and $\mathrm{C}$ are probably not as friend as with the common friend. This logic looses information in this context.

The Lukasiewicz logic uses the Lukasiewicz t-norm, Schweizer \& Sklar (2005), defined by $\mathrm{W}(\mathrm{a}, \mathrm{b})=\max (0, \mathrm{a}+\mathrm{b}-1)$. It works well when the common friend is a good friend of both, but it infers no friendship when the common friend is not a very good friend of both individuals.

So, the best logic to use to infer friendship is the Product logic (using the product t-norm). It works well for high and low friendship with a common friend, and it does not loose information. For example, if we have a common friend with degree $1 / 2$, the product logic would decide that we are friends with degree $1 / 4$, which makes more sense in human reasoning than infering a friendship degree of $1 / 2$ (Zadeh's logic) or 0 (Lukasiewicz logic). In the classical world, with only 0 and 1 values, all the logics, and transitivity closure are the same, but when we dial with the uncertainty of friendship, the product logic seems to be the most similar to human reasoning.

Another approach not referred here is to take into account the space and time dimensions. Even though space is implicitly covered when we let an agent to communicate only with its nearby ones, we ignore if an agent is closer than other: geography. This could be seen as another fuzzy relation, where 1 is closest neighbor, and 0 not known at all. About timing, it must be said that all the definitions here should be taken into account, because in the simulated system continuous time does not exist: time is discretised in time steps. This way, all the operations require a step of time. For example, a right way would be: $\mathrm{X}^{s+1}(\mathrm{Ind}):=\mathrm{X}^{s}$ (Ind) $+\Delta^{X, s}($ Ind) where "s" is the number of time steps. 


\section{Results and Conclusions}

The motivation for this work is the observation that certain characteristics of social systems are poorly modeled with crisp attributes. This has been illustrated with a concrete agent based system for the analysis of the evolution of values in a society, starting from a previous model of Pavón et al. (2008). Abstractions for the agent model can oversimplify the view of the reality, and complicate the social scientist to interpret results in the same terms in which the survey data or other observations are expressed. Furthermore, some simplifications produce divergences of results with respect to survey data. The use of fuzzy logic can improve agent models that get closer to reality. This has been explored in five aspects: relationships among agents, some variable attributes that determine agent states, functions of similarity, evolution of agent states, and inheritance.

Some aspects are appropriate for fuzzification, such as the friendship relation, but not for all, such as the family relation. Also, the compatibility concept for matchmaking, with all the restrictions, has needed changes in the similarity algorithm to a fuzzy version. Besides, we extracted new knowledge through the T-transitive closure. At the moment this work is not implementing the fuzzy influence, the fuzzy inheritance, and the fuzzy states. Anyway, with the actual changes, the similarity operation has improved substancially its accuracy; the couples found are more "logical" (reaching a $22 \%$ of improvement), due to two facts: agents know more people than before thanks to the closure, and the cited improvement in the measure of similarity; and the friendship is more realistic: instead of being boolean, it is graded, and it considers more knowledge thanks to the T-transitive closure. For more information about this work and a deeper analysis of its results, including graphs and tables of results, please refer to the works of Hassan, Salgado \& Pavon (2008).

The proposed fuzzification can be applied to other ABSS systems. For instance, the example has shown how to fuzzify relations that determine agents' interactions, even mixing fuzzy and crisp values (like in the case of "influence" interaction). Also, agents' attributes can be defined in terms of fuzzy sets. Context-dependant functions, like inheritance, were modelled too, as well as a typical fuzzy similarity operation. "Life states" of agents are frequent in systems that evolve over time, especially in task solving environment. Sometimes, it is convenient to fuzzify those states. Finally, a global fuzzy operation over all the agents was defined (the Ttransitive closure) on a fuzzy relation (friendship) to make inference with coherent results. Other operations could be needed and applied in a similar way.

In order to adapt these definitions to other systems, new constraints that suit context needs could be introduced. Fuzzy logic facilitates modelling to domain experts, because the linguistic terms can be easily transformed into fuzzy sets. Of course, there will be parts of an ABSS system that cannot be (or just we do not want to) fuzzified, but we think that fuzzy agents in social simulation can be extremely useful.

Future lines of research include empowering inference and approximate reasoning using fuzzy sets. Fuzzy implications represent a tool with great potential, and have solid foundations because they generalize the classic logical implication. As an interesting path to explore, their only special need is that the social scientist has 
to decide rules from his knowledge, and operators that work well in each context should be selected.

\section{Acknowledgements}

This work has been performed in the context of the projects "Métodos y herramientas para modelado de sistemas multiagente", and "Modelos de Representación, Agregación y Clasificación para la Toma de Decisiones con Información Imprecisa", supported by Spanish Council for Science and Technology, with grants TIN200508501-C03-01 and TIN2006-06190 respectively. Also, the authors would like to acknowledge the many helpful suggestions of two anonymous reviewers.

\section{References}

Axelrod, R. (1997), 'Advancing the art of simulation in the social sciences', Complexity 3, 16-22.

Carbó, J., Molina, J. M. \& Dávila, J. (2005), 'Fuzzy referral based cooperation in social networks of agents', AI Commun. 18, 1-13.

Conte, R. \& Paolucci, M. (2001), 'Intelligent social learning', Journal of Artificial Societies and Social Simulation 3.

URL: http://jasss.soc.surrey.ac.uk/4/1/3.html

European Values Survey (2000).

URL: $h t t p: / / w w w . e u r o p e a n v a l u e s . n l$

Garmendia, L., del Campo, C., Cubillo, S. \& Salvador, A. (1999), 'A method to make some fuzzy relations t-transitive', International Journal of Intelligent Systems 14, $873-882$.

Gilbert, N. \& Troitzsch, K. G. (1999), Simulation for the Social Scientist, 1 edn, Open University Press.

Hassan, S., Antunes, L., Pavon, J. \& Gilbert, N. (2008), Stepping on earth: A roadmap for data-driven agent-based modelling, in 'Fifth Conference of the European Social Simulation Association (ESSA08)', Brescia, Italy.

Hassan, S., Salgado, M. \& Pavon, J. (2008), Friends forever: Social relationships with a fuzzy agent-based model, in 'Hybrid Artificial Intelligence Systems 2008 (HAIS08)', Burgos.

Klement, A. E. P., Mesiar, R. \& Pap, E. (2000), Triangular Norms, Springer.

Menendez, M. A. (2004), Cambio cultural y cambio religioso. Tendencias y formas de religiosidad en la Espa $\backslash$ na de fin de siglo, Ed Complutense. Servicio de Publicaciones, Madrid.

Naessens, H., Meyer, H. D. \& Baets, B. D. (2002), 'Algorithms for the computation of t-transitive closures', Fuzzy Systems, IEEE Transactions on 10, 541-551. 
Norris, A. P. \& Inglehart, R. (2004), Sacred and Secular, Cambridge University Press, Cambridge.

Pavón, J., Arroyo, M., Hassan, S. \& Sansores, C. (2008), 'Agent-based modelling and simulation for the analysis of social patterns', Pattern Recogn. Lett. 29, 10391048 .

Repast (2008).

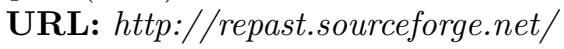

Schweizer, B. \& Sklar, A. (2005), Probabilistic Metric Spaces, Dover Publications.

Valverde, L. (1984), 'On the structure of f-indistinguishability operators'.

Wolfram, S. (2002), A New Kind of Science, 1 edn, Wolfram Media.

Yager, R. R. (1993), 'Families of owa operators', Fuzzy Sets Syst. 59, 125-148.

Zadeh, L. A. (1965), 'Fuzzy sets', Information and Control 8, 338-353.

Zadeh, L. A. (1971), 'Similarity relations and fuzzy orderings', Information Science 3, 177-200. 